role in the study design, data collection and analysis, decision to publish, or preparation of the manuscript. The authors declare that they have no competing interests.

\section{PRELIMINARY RESULTS OF ANLOTINIB AND NIRAPARIB DUAL THERAPY EVALUATION IN PLATINUM-RESISTANT RECURRENT OVARIAN CANCER (ANNIE): A MULTICENTER, SINGLE-ARM, PHASE 2 TRIAL}

${ }^{1}$ Guochen Liu, ${ }^{1}$ Jihong Liu, ${ }^{2}$ Bingna Xian, ${ }^{3}$ Yanling Feng, ${ }^{3}$ Qidan Huang. ${ }^{1}$ Sun Yat-Sen University Cancer Center; Department of Gynecologic Oncology; ${ }^{2}$ Sun Yat-Sen University Cancer Center; ${ }^{3}$ Department of Gynecologic Oncology

\subsection{6/ijgc-2020-ESG0.105}

Introduction/Background Patients with platinum-resistant ovarian cancer have a poor prognosis. Effective treatment options for these patients are limited. In this study (ANNIE), we evaluate the activity of niraparib combined with anlotinib in patients with platinum resistant recurrent ovarian carcinoma.

Methodology The ANNIE trial was a multicentre, single-arm, phase 2 study that evaluated the safety and activity of niraparib combined with anlotinib in patients $(\geq 18 \& \leq 70$ years) with recurrent ovarian epithelial, fallopian tube, or primary peritoneal cancer cancer whose disease recurred in less than 6 months after the last administered platinum therapy. Patients received oral niraparib $300 \mathrm{mg} / 200 \mathrm{mg}$ once daily continuously and anlotinib 12 mg on day 1-14 of each 21-day cycle thereafter until disease progression or intolerable toxicity. The primary objective was to assess objective response rate (ORR; complete plus partial responses). 40 cases are planned to be enrolled.

Results Between May 22, 2020 and August 3, 2020, we enrolled 9 patients (median age, 54 years [range, 44-64 years]). Patients had received a median of five (range, 2-8) previous lines of therapy. All but one (voluntarily withdrew) of the patients were still on treatment, the longest has been taking medication for more than 4 cycles. Three patients underwent imaging evaluation, including 1 confirmed complete responses, 1 with confirmed partial responses, 1 with stable disease. No drug-related grade 3 or worse treatment-emergent adverse events were detected, the most common treatment emergent adverse events were hypertension (5 of 9 patients), hand-foot skin reaction (4 of 9 patients), hoarseness (4 of 9 patients). Enrollment was ongoing so far.

Conclusion It seems niraparib in combination with anlotinib is tolerable, with promising antitumor activity for patients with platinum resistant recurrent ovarian cancer. Besides, we observed unusual safety signals in the combination (more hoarseness and less haematological toxicities). The conclusion can be clarified after the research is completed.

Disclosures Trial registration ClinicalTrials. gov identifier: NCT04376073, Funding: Zai Lab, The authors declare no conflicts of interest.

\section{\begin{tabular}{l|l}
247 & OPTIMISING PREDICTION ACCURACY OF COMPLETE
\end{tabular} CYTOREDUCTION FOR HIGH GRADE SEROUS ADVANCED OVARIAN CANCER PATIENTS USING NEAREST-NEIGHBOR MODELS}

Alexandros Laios, Yong Tan, Richard Hutson, George Theophilou, Diederick Dejong. St James's University Hospital; Leeds Teaching Hospitals; Gynaecologic Oncology
Introduction/Background The foundation of modern ovarian cancer care is cytoreductive surgery to remove all macroscopic disease (R0). Identification of R0 resection patients may help individualise treatment. Machine learning and AI have been shown to be effective systems for classification and prediction. For a disease as heterogenous as ovarian cancer, they could potentially outperform conventional predictive algorithms for routine clinical use. We investigated the performance of an $\mathrm{AI}$ system, the k-nearest neighbor (k-NN) classifier to predict R0, comparing it with logistic regression.

Methodolog A cohort of patients diagnosed with high grade serous advanced ovarian, tubal and primary peritoneal cancer (HGSOC), undergoing surgical cytoreduction from 20152019, was selected from the ovarian database. Performance variables included age, BMI, Charlson Comorbidity Index (CCI), timing of surgery, surgical complexity and disease scores. The k-NN algorithm classified R0 vs non-R0 patients using 3-20 nearest neighbors. Prediction accuracy was estimated as percentage of observations in the training set correctly classified. The relative importance of the selected variables was quantified by calculating the prediction accuracy/ error rate in relation to the number of predictors included in the models.

Results 154 patients were identified, with mean age of $64.4+$ 10.5 yrs, BMI of $27.2+5.8$ and mean SCS of $3+1$ (1-8). Complete and optimal cytoreduction was achieved in $62 \%$ and $88 \%$ patients. The mean predictive accuracy was $66 \%$ compared to $63.4 \%$ by logistic regression. R0 resection prediction of true negatives was as high as $90 \%$ using $\mathrm{k}=20$ neighbors. From the variables tested to contribute in R0 prediction, only disease score was statistically significant $(\mathrm{p}=0.0006)$. For a given neighborhood size $\mathrm{k}=15$, $\mathrm{R} 0$ resection was best

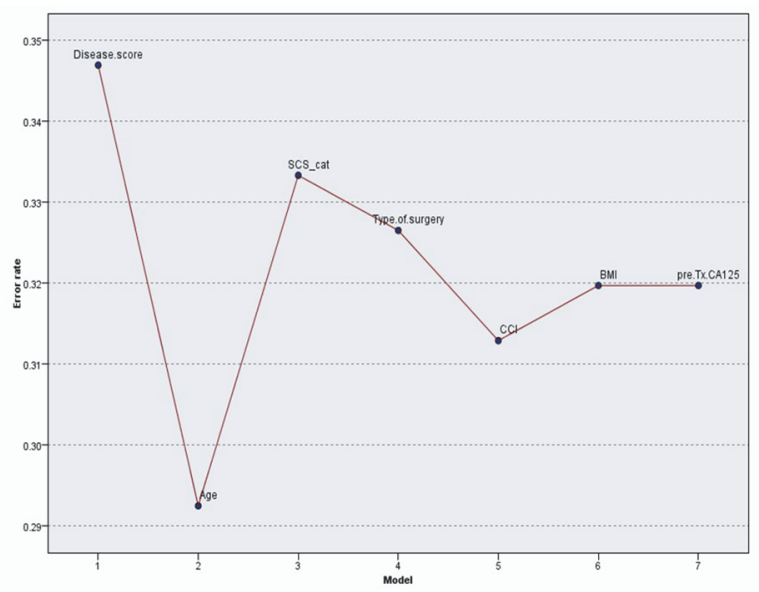

Abstract 247 Figure 1

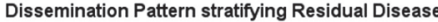

R1 R0

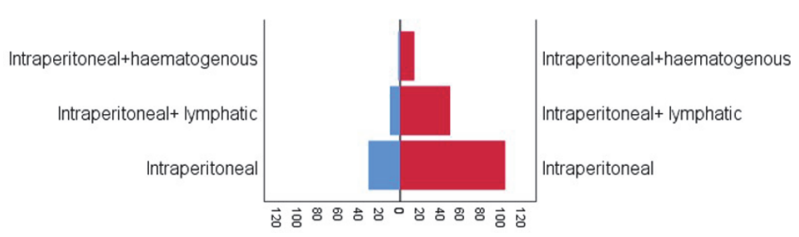

Abstract 250 Figure 1 